\title{
Software-Defined Underwater Acoustic Modems: Historical Review and the NILUS Approach
}

\author{
Henry Dol, Paolo Casari, Senior Member, IEEE, Timo van der Zwan, Roald
}

Otnes, Senior Member, IEEE

\begin{abstract}
Flexible/adaptive acoustic modems that are reprogrammable/reconfigurable at all layers of the communication stack, either by a user or by means of autonomous decisions, are considered as an important enabler for interoperability and cognitive networking in the underwater domain. In this paper, we review the existing literature on software-defined acoustic modems for underwater communications and networking, considering past and ongoing academic efforts, as well as industrial developments and European collaborations centered on software-defined modem structures and functionalities. We then zoom in on relevant R\&D efforts currently taking place in a Netherlands-Norway defense cooperation, targeting the design of a software-defined modem for the NILUS MK 2 sensor node. This modem is built using general-purpose computing architectures running an open-source operating system and tools, thereby making a further step towards software-defined open-architecture underwater acoustic modems. The first field tests of the NILUS MK 2 node in multihop underwater acoustic networks are presented, showing satisfactory performance in shallow and open waters.
\end{abstract}

\section{Index Terms}

Software-defined modems, underwater acoustic communications, underwater wireless networks, open architecture, sea trials.

Henry Dol (e-mail: henry.dol@tno.nl) and Timo van der Zwan are with TNO, P.O. Box 96864, NL-2509JG, The Hague, The Netherlands. Paolo Casari (e-mail: paolo.casari@imdea.org) is with the IMDEA Networks Institute, Madrid, Spain. Roald Otnes (e-mail: Roald.Otnes@ffi.no) is with FFI, P.O. Box 115, NO-3191 Horten, Norway. 


\section{INTRODUCTION}

Historically, the development of reconfigurable, reprogrammable and software-defined underwater acoustic modems has been driven by universities and research institutes, mainly because of the flexibility required by scientific experiments on underwater communications and networks. More recently, lacking standardization of underwater communication protocols, the modem industry has been gradually opening their commercial off-the-shelf (COTS) systems to the (skilled) end-user as well. This provides better opportunities for interoperability, among modems made by different manufacturers, and adaptability, i.e., being able to switch protocols or parameter settings depending on an evolving environment or on changing application requirements. However, the reconfiguration/reprogramming options for commercial modems today are typically limited, and any change requires a thorough knowledge of the specific hardware and software architecture. Therefore, an interesting next step would be the introduction of open-architecture/open-source modems, fostering the involvement and support of a global user community. This would boost the establishment of standards and, more importantly, their acceptance. Modem manufacturers could still make a profitable business, but their business model would change by focusing more on efficient hardware and proprietary high-performance algorithms.

In this paper (Section II), we provide a review of past and current efforts towards the construction of reconfigurable/reprogrammable and software-defined modems, listing relevant hardware and software specifications when available. We proceed by detailing a joint effort of the Netherlands Organisation for Applied Scientific Research (TNO) and the Norwegian Defence Research Establishment (FFI) towards the development of a fully flexible and high-level programmable modem, which still complies with operational requirements in terms of processing power, size and endurance (Section III). Since this software-defined modem runs a Linux operating system, the Netherlands-Norway defense cooperation will contribute to the development of a Software-Defined Open-Architecture Modem (SDOAM). This and other concluding remarks are provided in Section IV. 


\section{REVIEW OF RECONFIGURABLE, REPROGRAMMABLE AND SOFTWARE-DEFINED}

\section{ACOUSTIC MODEMS}

Following developments in terrestrial radio systems, an upcoming concept for underwater acoustic modems is software-defined, possibly open-architecture, reconfigurable/user-programmable modems. The current state of these developments, which mainly takes place in a non-commercial setting, is described in this section. The research on reconfigurable acoustic modems is mainly driven by the need for an adaptive platform, whose characteristics (in terms of physical-layer schemes, or network protocols, or both) can be changed to suit the need of different experiments/scenarios.

The first commercial modems to be available on the market basically served as serial link emulators, providing remote data telemetry via acoustic communications. Modem systems were mostly opaque to the end-user, as their physical-layer (PHY) algorithms and bit-stream formats were hardcoded in the modem firmware. Moreover, in some cases their network (NET) protocol implementations included some form of preliminary or periodic messaging which was also not controllable/reconfigurable. For these reasons, commercial off-the-shelf equipment was usually transparent to the application and therefore not very well suited to research efforts.

More recent modems include at least some form of reconfigurability. A reconfigurable modem gives the user the chance to decide on some parameters by issuing commands that are recognized by the modem's firmware. On-the-fly changes of the digital modulation's bit rate and coding scheme at the PHY layer are a typical example of this reconfiguration capability. With the development of underwater modem hardware and firmware for research projects in academia, the options available for the users have been broadened by reprogrammability. Reprogrammable modems introduce the freedom to replace one or more of the main communication blocks including, e.g., complete modulation schemes, signal processing algorithms or network-layer protocols. While this possibility clearly represents a valuable tool, reprogramming a modem

often requires additional skills such as, e.g., the programming of digital signal processors (DSPs) 
or field-programmable gate arrays (FPGAs) for PHY signal processing schemes, or the use of specific or uncommon programming languages for the network protocols stack. Software-defined modems (sometimes also briefly called softmodems) are a specific case of reprogrammable modems where the whole transmitter- and receiver-side processing chains are programmed in software. The recent development of open-source and open-architecture modems is a significant step towards making modem architectures more accessible to the end-user. With open-source modems, users can refer to well-defined programming examples and templates to introduce their own functionalities in the PHY or NET layers, or both. Open-architecture modems make this concept even more accessible by running software and algorithms on standard computing devices, whose architecture is well known. A relevant example is the use of general-purpose (GP) processors to replace specialized hardware such as DSPs.

Jones [1] was one of the original advocates in the open literature for software- and cognitiveradio concepts in underwater systems. She advocated methods for changing the modulation scheme without modifying the whole system architecture. Depending on the degree of reconfigurability/reprogrammability sought, research projects on reconfigurable modems may focus on the flexibility of the physical layer, of the network protocols, or of both. In the following, we will describe the relevant research projects on reconfigurable modems that we are aware of. The review is divided into past (mainly USA) academic efforts (2006-2010, Section II-A), ongoing academic efforts (1999-, Section II-B), industrial developments (2010-, Section II-C) and European collaborations (2005-, Section II-D). Section II-E summarizes the findings of this survey. For the reader's reference, the characteristics of the surveyed modems are summarized in Table I along with reported results of laboratory and/or field experiments for each modem.

\section{A. Past academic efforts}

Between 2006 and 2010, seven academic projects on reconfigurable and reprogrammable underwater acoustic modems have been running, mostly in the USA, which have set the first milestone for the development of software-defined (open-architecture) underwater acoustic modems. 
1) MIT Sea Grant's rModem (USA, 2006-2008): MIT's rModem project dates back to the years 2006-2008. The project aimed at building a piece of hardware which was reprogrammable in terms of physical-layer (PHY) techniques for communication and signaling, and that could provide typical PHY-related measurements (e.g., reliability) and services (e.g., high-resolution power control) [2], which were not typically available in off-the-shelf modems, not even Woods Hole Oceanographic Institution's MicroModem [3] (Section II-B1), that was the most reconfigurable underwater modem available at the time. The rModem is composed of one motherboard and one daughter card. Its main features include 4 configurable I/O channels suitable for multipleinput multiple-output (MIMO) algorithms, analog anti-aliasing filters, an on-board FPGA and a $300 \mathrm{MHz}$ floating-point DSP with $32 \mathrm{MB}$ SDRAM and $32 \mathrm{MB}$ flash memory [2]. The daughter card hosts signal conditioning and amplification stages, and is detached from the motherboard to improve the platform flexibility whenever, e.g., the transducer connected to the modem has to be changed. The FPGA hardware is fast enough to process any signal in the $1-100 \mathrm{kHz}$ band. At the time, [2] reported successful communication experiments in the 9-14 kHz band using a Teledyne AT-408 transducer.

The hardware can be operated in real-time (if the DSP supports the complexity of receiverside signal processing) or in pseudo real-time (whereby signal samples are stored before being processed by the DSP, therefore introducing some processing delay). A specific implementation of a transmitter and a receiver on the rModem is documented in [4], where successful reception of Quadrature Phase-Shift Keying (QPSK) signals is reported at 550 bit/s within a tank, using a carrier frequency of $12 \mathrm{kHz}$. An adaptive decision-feedback equalizer (DFE) is employed for phase-coherent reception, which embeds the recursive least squares (RLS) algorithm and a phase-locked loop. A convolutional code is used for error correction.

2) UCD/UCI/IST Tmote Invent-based Underwater Node (Ireland/USA/Portugal, 2006-2007): The first known design of a low-cost, software-defined underwater acoustic modem has been carried out in the context of a collaboration between University College Dublin (UCD), University of California Irvine (UCI) and Instituto Superior Técnico (IST), Lisbon [5], [6]. The objective was 
to create an inexpensive platform for underwater communications that relied on integrated lowcost components to carry out modulation and demodulation tasks. After a test with laptops [5], Moteiv's Tmote Invent/Sky platform was chosen [6] and adapted for underwater usage via waterproof latex membranes. The devices rely on a microphone sensitive to the $0.1-20 \mathrm{kHz}$ band, and on a speaker working in the $0.4-20 \mathrm{kHz}$ band, which are employed as acoustic receiver and transmitter, respectively. A Frequency-Shift Keying (FSK) scheme was designed by employing eight frequency bands that exhibited good performance at several distances between 1 and $13 \mathrm{~m}$ in preliminary performance measurements. The modulation and demodulation chains are programmed in software within the node's microcontroller. The system achieves error-free communication at $24 \mathrm{bit} / \mathrm{s}$ (and possibly up to $48 \mathrm{bit} / \mathrm{s}$ ) up to a distance of $17 \mathrm{~m}$. As the Tmote Invent is a very resource-constrained platform, it is not clear whether its microcontroller could host both PHY and NET code simultaneously. All the same, the result marks an achievement in terms of software-defined low-cost modems.

3) MIT CSAIL's AquaNodes (USA, 2007): In MIT's AquaNodes project, the physical-layer implementation is fixed, but the user is free to reprogram network protocols [7]. AquaNodes can communicate by means of either acoustic or optical signals, although the latter can only be operated within a limited distance of $2 \mathrm{~m}$ and when the transceivers are aligned within a tolerance of $90^{\circ}$. The AquaNodes embed an ATmega128 microcontroller, $128 \mathrm{kB}$ of flash memory, $4 \mathrm{kB}$ of RAM and $512 \mathrm{kB}$ of external storage.

Acoustic communications are realized via an FSK modulation scheme at a carrier frequency of $30 \mathrm{kHz}$. Data rates of $330 \mathrm{bit} / \mathrm{s}$ up to a range of $400 \mathrm{~m}$ are reported. Optical communications are obtained by interfacing the motherboard to a special-purpose board through a serial link. The optical board generates signals at a wavelength of $532 \mathrm{~nm}$, and a communication range of $2.2 \mathrm{~m}$ (up to $8 \mathrm{~m}$ with beam-narrowing lenses and precise pointing). The maximum data rate reported is $320 \mathrm{kbit} / \mathrm{s}$. A Lithium-ion battery of $56 \mathrm{Wh}$ provides sufficient power supply for one year (1-2 weeks) at minimum (maximum) duty cycle. The reconfiguration of the networking components of the AquaNodes is achieved through a communication channel to the modems (a 
wired serial cable, a Bluetooth connection, acoustic as well as optical wireless communications). In [7], a time-division multiple-access (TDMA) channel access system with self-synchronization capabilities is employed.

4) UCLA's UANT (USA, 2009): The Underwater Acoustic Networking PlaTform (UANT) was probably the first software-defined, open-source underwater platform prototype specifically designed to be reprogrammable at both the physical layer and the network layer [8]. PHY reprogramming is achieved via GNUradio (a widely adopted platform in the software-defined radio community) whereas NET reprogrammability is provided by TinyOS (a standard solution for programming network protocols in wireless sensor networks). Since GNUradio and TinyOS are open-source / open-architecture developments (for the terrestrial radio environment), the UANT project was well ahead of its time.

GNUradio is run on a Universal Software Radio Peripheral (USRP) that, albeit more expensive than a low-cost audio board, provides more processing capabilities via dedicated hardware. With the choice of GNUradio, many of the signal processing blocks already programmed for the radio world can be ported to underwater communications, and can be assembled to (re)configure basic and advanced modulation schemes. Specific changes to GNUradio include allowing custom medium access control (MAC) protocols, setting the bit rate, and making the traffic source and sink TinyOS-based so that traffic can be generated and processed within the networking components of the UANT. The changes have been kept limited in order to maximize the support for future expansions to GNUradio's digital communication blocks.

GNUradio has been interfaced with TOSSIM, the simulator of TinyOS. This makes it possible to implement network protocols with a tool commonly employed for programming wireless sensor networks. The interface intercepts the packets coming from the transducer and passes them to a MAC protocol in TOSSIM's stack. From there, further network protocol processing can start. The use of TOSSIM also enables application-level (APP) reprogramming. UANT runs on the following hardware [8]: Intel Core 2 Quad Q8200 processor, 4 GB RAM, USRP LowFrequency TX and RX daughter cards (0-50 MHz), custom pre-amplifier board, and RESON 
TC4013 hydrophone (RX bandwidth $0.1-175 \mathrm{kHz}$ ). The extension of the UANT with a channel allocation protocol and a time synchronization protocol is described in [9].

5) UCSD's low-cost modem (USA, 2010): As experimentation costs represent a heavy burden for the budget of academic underwater networking projects, some underwater acoustic modems were specifically designed to be both software-defined and low-cost.

UCSD's low-cost modem design is tailored to extract the most from a \$10 Steminc SMC26D22H13 ceramic ring transducer [10]. This is achieved via a highly linear $75 \%$-efficient mixed-class $\mathrm{AB}+\mathrm{D}$ power amplifier, a high-gain receiver, and output power control. The transmittter- and receiver-side signal processing are hosted on an FPGA mounted on a DINI Group DNMEG AD-DA evaluation board. The resulting system is based on a 200 bit/s FSK modulation scheme working in the $32-38 \mathrm{kHz}$ band, at a final estimated cost of $\sim \$ 600$, when produced in large volumes. Controlled tests in Mission Bay, San Diego, CA, confirmed the capability to detect signals from a distance of $350 \mathrm{~m}$.

6) UNSW's high-rate modem (Australia, 2010): Nowsheen et al. detail a preliminary implementation of a software-defined modem consisting of a BPSK modulation-demodulation chain programmed on an FPGA [11], [12]. Most of the code has been written in Matlab and tested on recorded waveforms before actually implementing the same functions on the FPGA. Successful open water tests are reported to have achieved a bit rate of $80 \mathrm{kbit} / \mathrm{s}$ using a carrier frequency of $800 \mathrm{kHz}$ at a distance of up to $50 \mathrm{~m}$. The same modem technology has been tested over a set of different synthetic (i.e., modelled) channels. The rationale and results of these tests are reported in [13].

7) CAS software-defined modem (China, 2010): Li and Huang designed a software-defined acoustic modem around a core Blackfin 561 DSP module [14]. The modem is programmed with different modulation schemes and coding rates, which are tested in a sea trial over a point-to-point link. A bit rate of up to $8 \mathrm{kbit} / \mathrm{s}$ over a range of $8 \mathrm{~km}$ is reported to be achieved using the QPSK modulation and a convolutional channel code within a bandwidth of $10 \mathrm{kHz}$ at a carrier frequency of $25 \mathrm{kHz}$. A higher-rate OFDM modulation scheme using turbo channel coding is reported 
to have achieved $20 \mathrm{kbit} / \mathrm{s}$ over a range of $1 \mathrm{~km}$. Network tests with four nodes employing a carrier-sense multiple access/collision avoidance (CSMA/CA) MAC protocol are also mentioned. It remains unclear whether the network protocols can be reconfigured/reprogrammed and on which system component they are run.

\section{B. Ongoing academic efforts}

Since 1999, four USA academic efforts on software-defined underwater acoustic modems are still active, of which one is being sold not-for-profit (Section II-B1), a second one is now generating a commercial spin-off (Section II-B2), a third one is still functional as part of an operational trial system (Section II-B3), and the fourth one is a more recent development that has already seen a number of tests in the water (Section II-B11). Since 2012, an important contribution to the development of software-defined underwater acoustic modems comes from Singapore (Section II-B5). More recently, an Italian effort targeted the easy reconfiguration of the modem's PHY and was used for double-PHY tests in the water (Section II-B8). Around the same time, a doubly-reprogrammable UK modem seeking robustness in harsh environments has been proposed (Section II-B10). All three academic developments (from Singapore, Italy and UK) have generated a commercial spin-off. Furthermore, two Chinese academic efforts are being proposed, based on different types of reprogrammable platforms (Section II-B7 and II-B9). Finally, two academic efforts also worth mentioning focus on low power (Spain, Section II-B4) and low cost (Pakistan, Section II-B6), respectively.

1) WHOI's MicroModem (USA, 1999-): The MicroModem of the Woods Hole Oceanographic Institution (WHOI) [3] has been an important communication platform since 1999 [15, Table 1], equipped with multichannel PSK (subject to US export restrictions) and lower-rate FrequencyHopped FSK (FH-FSK, available for export) modulations. The MicroModem was licensed non-exclusively to Hydroid/Kongsberg to be mounted on the REMUS AUVs (Autonomous Underwater Vehicles). Since about 2010, the MicroModem has been superseded by a more powerful version [15]. The proprietary physical-layer schemes are largely opaque to the end-user 
with limited reconfiguration possibilities, and user-reprogrammability is not explicitly supported. However, the technical details about the modem architecture as provided on the WHOI MicroModem website [16] may allow independent developers to implement their own modulations and network stack on the DSP system, possibly partly on the optional Gumstix expansion board. Compared to the original MicroModem, the newer version embeds a more powerful fixed-point Analog Devices Blackfin DSP, a data-acquisition FPGA and increased memory sizes [15]. The PSK scheme runs separately on a floating-point coprocessor, and the multichannel variant uses an additional analog interface board. The coprocessor tasks can in principle be taken over by the Blackfin DSP.

2) University of Connecticut's OFDM modem (USA, 2007-): The Underwater Sensor Network (UWSN) lab at the University of Connecticut has been actively developing an underwater modem based on MIMO Orthogonal Frequency-Division Multiplexing (OFDM) [17]. A real-time DSP implementation of both a single input-single output (SISO) and a MIMO version of the modem is detailed in [18]. In [17], the authors report results based on channel recordings from the RACE'08 campaign. QPSK up to 64-QAM with two transmitters, QPSK up to 16-QAM with three transmitters, and QPSK up to 8-QAM with four transmitters are used with a bandwidth of $4.8 \mathrm{kHz}$. The achievement of a $125.7 \mathrm{kbit} / \mathrm{s}$ data rate with two transmitters, 16-QAM modulation, rate-1/2 coding, and a bandwidth of $62.5 \mathrm{kHz}$ is reported related to a second set of experiments in 2008 .

The implementation detailed in [18] demonstrates real-time capabilities with both a floatingpoint (TI TMS320 C6713) and a fixed-point (TI TMS320 C6416) DSP, achieving data rates of 3.2 and $6.4 \mathrm{kbit} / \mathrm{s}$, with a SISO and a $2 \times 2 \mathrm{MIMO}$ modem (where the notation indicates the number of transmitter-side and receiver-side elements, respectively). A QPSK modulation scheme and a rate-1/2 convolutional channel code within a $6 \mathrm{kHz}$ bandwidth were employed. The use of a low-density parity-check (LDPC) code is also demonstrated to be possible in real time.

Since 2012, the OFDM modem efforts of the University of Connecticut have generated 
a commercial spin-off called AquaSeNT. The final design of the modem includes a carrier frequency of $24 \mathrm{kHz}$, a bandwidth of $6 \mathrm{kHz}$, and five transmission modes that mix different subcarrier modulation schemes (BPSK, QPSK and 16-QAM) and different code rates (1/2 and 3/4) at a maximum bit rate of $9 \mathrm{kbit} / \mathrm{s}$ [19, Ch. 18]. The different modulation and coding schemes are tested via simulations and a sea trial in [20]. A framework called SeaLinx implements networking components as operating system processes, which inherently supports real-time operations [21], [22].

3) UCSD Scripps' SDAM (USA, 2010-): The Software-Defined Acoustic Modem (SDAM) development by the Scripps Institution of Oceanography at UCSD is mainly aimed at multichannel and MIMO communications in the $10-32 \mathrm{kHz}$ band [23] . Real-time acquisition and processing for 8 channels is obtained via an NI-7833R board (100 kHz sampling rate at 16 bits per sample). A CPU board (CP306, containing a $1.4 \mathrm{GHz}$ Pentium-M on a single PCI board, 1 GB RAM, USB 2.0, $1 \mathrm{Gbit} / \mathrm{s}$ LAN) can be programmed to handle non-intensive computations and networkingrelated operations. A storage capacity of 2 GB, a precision GPS and a high-efficiency custom Pulse Width Modulation (PWM) power amplifier complement the board electronics. Hard disks provide storage capacity for both the operating system of the board and real-time data acquisition. The Source-Receive Array (SRA) is composed of 8 ITC-1001 spherical transducers.

UCSD's SDAM is doubly reprogrammable: the FPGA on the digital acquisition board can be leveraged for upshifting and resampling, as well as for more complex baseband processing, while the general-purpose CPU can run network protocols and higher-level functions.

4) University of Valencia's low-power ITACA modem (Spain, 2011-): The idea behind the ITACA low-power modem design is that the microcontroller should be relieved from burdensome tasks such as continuous low-pass filtering [24]. This pushed forward the design of a very-low-power modem, employing a Humminbird XP 920 transducer resonating at $85 \mathrm{kHz}$, and transmitting via a coherent FSK scheme (1 kbit/s over a bandwidth of $1 \mathrm{kHz})$ implemented on a C8051f360 Silabs microcontroller. A custom push-pull class-B-based digitally controlled power amplifier also contributes to the low power consumption. Experiments in 
Murcia, Spain, proved communication capabilities over $100 \mathrm{~m}$-long horizontal channels. Later improvements [25] include a longer communication range, an RFID-based low-power wake-up module, and in-hardware clear-channel assessment.

5) National University of Singapore's UNET-2 modem (Singapore, 2012-): The UNET-2 modem is a research platform for underwater communications that offers different types of reprogrammability [26]. The modem builds on a previous development at the National University of Singapore's (NUS) Acoustic Research Laboratory (ARL), namely the so-called ARL modem. This modem employs an OFDM modulation scheme to communicate up to a declared distance of $3 \mathrm{~km}$ and $7.5 \mathrm{kbit} / \mathrm{s}$ at a $31.25 \mathrm{kHz}$ carrier frequency with $12.5 \mathrm{kHz}$ of bandwidth [27]. The UNET-2 modem extends this design by supporting carrier frequencies up to $78 \mathrm{kHz}$, and a modulation bandwidth up to $2 / 3$ of the chosen carrier frequency. The preconfigured modulation schemes consist of an incoherent and a coherent OFDM scheme whose parameters can be dynamically controlled. The applied coding scheme and the source level of the modem can also be dynamically controlled.

The system is composed of a Toradex Collibri PXA270 single-board computer (SBC), an Analog Devices TigerSHARC TS201 DSP, and an Altera Stratix-III FPGA, where the FPGA carries out detection and passband processing, the DSP implements PHY processing and coding, and all NET layer processing resides on the SBC. The latter can be reprogrammed by implementing new Java Agent Development Framework (JADE) components, whereas PHY schemes need be programmed and cross-compiled for the TS201 DSP. The authors in [26] report in-water tests to showcase the reconfiguration capabilities of the modem and a link error control protocol.

6) FAST-NUCES' low-cost modem (Pakistan, 2012-): Ahmed et al. [28] detail a do-it-yourself (student) modem design that employs common off-the-shelf materials (e.g., car tweeters, vegetable oil, etc.), and reuses common computing equipment (e.g., laptops) to carry out signal processing tasks. The latter is achieved via the GNUradio software, plus an interface that makes it possible to attach custom network protocols to the communications framework. Tank tests demonstrated the feasibility of communications at a rate of 100 to $500 \mathrm{bit} / \mathrm{s}$ over distances up 
to $6 \mathrm{~m}$. Excluding the laptops, the cost of additional components ranges from approx. $\$ 25$ to $\$ 50$ if a high-performance ceramic tweeter is used to achieve a longer communication range.

7) Harbin IMMP platform (China, 2012-): Zhang et al. [29] propose to centralize the control of the different modem components into a management platform, which is built around an ARM 6410 processor. While the modem attached to the platform is not reprogrammable, the platform offers sufficient flexibility to (re)configure the modem, the storage, the wake/sleep subsystems, etc. The platform also offers facilities to program and implement network protocols. The implementation of the ALOHA, CSMA and TDMA MAC protocols is reported in [29] and is tested over a network of three nodes deployed in the Yellow Sea. The authors report successful communications over a distance of up to $30 \mathrm{~km}$.

8) University of Calabria and AppliCon's SeaModem (Italy, 2014-): The University of Calabria, Italy, in collaboration with their spin-off AppliCon, have been developing SeaModem, a low-cost underwater acoustic modem that supports both physical- and network-layer reprogrammability [30]. The modem operates in the $25-35 \mathrm{kHz}$ band, and is composed of a DSP board and an ARM-based BeagleBone board for general-purpose reprogrammability. A flash memory hosts the possible system configurations, including a read-only factory default and up to 4 rewritable configurations. Reconfigurable physical-layer parameters include the number of tones of the native M-FSK modulation (2, 4 or 8, corresponding to bit rates of 750, 1,500 and $2,250 \mathrm{bit} / \mathrm{s}$, respectively) and the gain of the class-D power amplifier, which outputs up to $40 \mathrm{~W}$ of power. A point-to-point link between two SeaModems has been preliminarily tested in a marina environment, where ranging estimations performed via the modem's two-way communication patterns were reported to achieve a relative error of about 0.5 to $3 \%$, depending on the actual range.

In [31], the authors present a more advanced configuration involving two separate physical layers, namely the SeaModem's native BFSK scheme and the JANUS FH-BFSK scheme [32]. The experiment involved three static underwater nodes and one unmanned surface vehicle (USV). The latter was instructed along a trajectory that sequentially led it within the coverage range of 
the underwater nodes. The USV would use JANUS to exchange first-contact information, and then switch to the proprietary BFSK physical layer of the SeaModem. JANUS was observed to be quite robust against communication errors, and for this reason it was also used as a means of remote control in case of unexpected behaviors. The SeaModem is reported to achieve a packet error rate below $10 \%$ at a distance below $400 \mathrm{~m}$, although it is not clear what packet size this figure applies to, and whether the difference between the native scheme and the JANUS scheme comes from the higher bit rate, the lack of selection diversity, convolutional coding and Viterbi decoding at the receiver, or from specific channel patterns. In any event, [31] presents the first preliminary experiment of a dual-PHY modem involving JANUS. The authors remark that the version of JANUS employed for this test is not fully compliant with the upcoming NATO standard in two respects: operational bandwidth (25-35 kHz instead of 9.44-13.6 kHz) and MAC (CSMA/CA with binary exponential backoff replaced by ALOHA).

9) Xi'an underwater acoustic modem (China, 2014-): Wang et al. [33] describe an underwater acoustic modem design based on the TI OMAP-L138 processor, which integrates C6000's DSP facilities and an ARM processor. The modem is the result of a collaboration between the Northwestern Polytechnical University of Xi' an and the China Ship Research and Development Academy. The authors implemented an OFDM modulation scheme with 512 QPSK-modulated subcarriers at a total bit rate of $4 \mathrm{kbit} / \mathrm{s}$. The connection between the modem hardware and a PC is required to feed the correct transmission and reception parameters to the DSP. The modem architecture has been tested using data recorded from a lake experiment.

10) Newcastle University and Blueprint Subsea's SeaTrac modem (UK, 2014-): Blueprint Subsea developed the SeaTrac modem based on front-end specifications by Newcastle University, UK, in the context of the EU-FP7 CADDY project [34]. The aim of the project is to provide reliable communication links among a diver, an AUV and an ASV (Autonomous Surface Vehicle). The acoustic front end operates in the $24-32 \mathrm{kHz}$ band, with a single transmitting piezoelectric transducer element and 4 smaller elements above, forming a 3D ultra-short baseline (USBL) array. Signal processing is implemented on the microcontroller embedded in the ARM Cortex 
M4 architecture. The modem supports $i$ ) a low-data rate mode (about $100 \mathrm{bit} / \mathrm{s}$ ), which involves up-sweeping and down-sweeping linear chirps that represent the ones and zeros of a binary digital signal, and $\mathrm{ii}$ ) an intermediate-data rate mode that achieves a link throughput up to $1.4 \mathrm{kbit} / \mathrm{s}$ via QPSK symbols spread by short chunks of a 8,191-point binary m-sequence (via direct-sequence spread spectrum, DSSS), block Doppler compensation and Reed-Solomon (RS) coding. The lowrate modulation is also leveraged on for USBL processing. The class-D amplifier of the modem outputs source levels of up to $176 \mathrm{~dB}$ re $1 \mu \mathrm{Pa}^{2} @ 1 \mathrm{~m}$. A network layer is not yet implemented as it is not needed for the short-range star-topology network of CADDY.

Tests carried out in marinas and pools featuring harsh multipath conditions showed the high robustness of the low-bit rate scheme and the good robustness of the medium-rate scheme, both in terms of error rate and in terms of range estimates. The modem described in [34] is not presented as a reprogrammable-by-design platform. In addition, it mounts some specialized hardware (e.g., four 16-bit analog-to-digital converters), and from the information in [34] and on the manufacturer's web site, it does not seem to operate on top of a general-purpose operating system (OS). However, the fact that the signal processing is carried out on an ARM Cortex architecture implies that changing the modulation scheme potentially just means running a different program on the processor. In addition, with sufficient memory available, the same architecture could double as a controller for the network protocols. The latter configuration is considered and proven by the NILUS modem, which works by leveraging on the components already available on a standard system-on-module board, and an open-source OS to drive the modem. The NILUS approach is presented in Section III.

11) University at Buffalo and Northeastern University's SEANet and SDAM prototypes (USA, 2015-): A collaboration between the University at Buffalo, NY, and the Northeastern University in Boston, MA, USA, led to the design of a software-defined acoustic networking framework [35] and of a reprogrammable acoustic modem [36] constructed using COTS components. In particular, the high-rate reprogrammable version of the software-defined acoustic modem (SDAM) detailed in [36], [37] is built around software-defined radio components. The system includes an 
USRP N210 based on the Xilinx Spartan 3A-DSP 3400 FPGA, with two daughter cards providing an operational frequency range of 0 to $30 \mathrm{MHz}$, which is adequate for the RESON TC4013 transducer (range from $1 \mathrm{~Hz}$ to $170 \mathrm{kHz}$ ). External pre-amplifier and power amplifier circuitry completes the setup. The baseband signal processing algorithms and the network protocols are executed on a host PC running software such as GNU Radio and Matlab, and are connected to the SDAM via a gigabit Ethernet link.

The system provides reconfigurability options that include switching the data and coding rate of the modulation scheme in use, as well as switching between different schemes. Adaptive mechanisms to switch between different PHY configurations have been implemented and tested both in a tank and in a lake experiment. The platform provides support for full PHY and network reprogrammability by acting on the software in the host PC and on the FPGA on-board the USRP, if needed. A low-end version is detailed and tested in a tank in [35], and incorporates the Teensy 3.1 development platform in place of the USRP N210.

The latest development of the platform, named SEANet-G3 [38], is based on a Xilinx Zynq Z-7020 System-on-Chip architecture incorporating a dual ARM Cortex-A9 (866 MHz) and an Avnet PicoZed XC7Z020-1CLG400 FPGA. Recent lake tests demonstrated that the platform can achieve a data rate of about $500 \mathrm{kbit} / \mathrm{s}$ over short-range horizontal links (e.g., $10 \mathrm{~m}$ ).

\section{Industrial developments}

Since 2010, several commercial modem manufacturers are making steps towards more reconfigurability and reprogrammability for the (skilled) end-user. This concerns two European companies (Germany), one Asian company (Singapore) and one USA-based company.

1) Develogic HAM series (Germany, 2010-): The first commercial underwater acoustic modem manufacturer that opened their system to the end-user is Develogic (Germany), by releasing their Hydro Acoustic Modem (HAM) series in 2010. Using a JTAG interface and Texas Instrument's Code Composer Studio, the TI DSP of the Develogic modem can be reprogrammed in $\mathrm{C} / \mathrm{C}++$ with another physical-layer protocol in a GNUradio-based framework (including a signal- 
processing library) [39]. The off-the-shelf HAM modems do not contain a network layer, but within a European collaboration (Section II-D3) it was shown that network-layer functionality can be successfully added to the Develogic modem by adding an additional external processor board.

2) EvoLogics WiSE series (Germany, 2012-): A few years later, EvoLogics released a COTS underwater acoustic modem, named WiSE [40] (White-line Science Edition), which embeds a network-development framework in a sandbox (including, e.g., a pre-installed $\mathrm{C} / \mathrm{C}++$ toolchain and interpreters for scripting languages). This enables support for network-protocol simulation and development packages such as ns2: as an example, the ns2/MIRACLE-based DESERT Underwater [41], [42] and SUNSET [43] frameworks leverage on this flexibility in order to drive EvoLogics modems. In addition to the above, EvoLogics modems provide a protocol called D-MAC [44], that can be exploited to access both the burst-data transmission and the instant-message schemes of the EvoLogics modems (Sweep-Spread Carrier modulation, S2C), as well as more advanced functions including positioning [45] and high-precision transmission timing.

More recently, EvoLogics has designed a framework for underwater networking and positioning called EviNS [46], which is written using the Erlang language. The objective of the framework is to provide lightweight concurrency, soft real-time operations, easier updates while the software is running and robustness against software errors. The latter is inherent to the Erlang philosophy, where different processes are isolated by design, such that software misbehaviors due to erroneous programming do not propagate to the whole system and can be easily recovered from.

3) Subnero's modem and UnetStack (Singapore, 2013-): In October 2013, NUS ARL and the Subnero company released v1.1.1 of UnetStack, an agent-oriented Java/Groovy implementation of an underwater networking stack [47]. Born from one of the first unified simulation and experimentation frameworks proposed and successfully demonstrated in water using the ARL modems [27], UnetStack was introduced and thoroughly discussed in [48], and was tested first 
on the NUS UNET-2 modems [26] (Section II-B5), on which the Subnero modem [49] is based. UnetStack makes it possible both to reprogram the network protocols and to reconfigure their parameters. Subnero's modems widen the reprogramming options to include physical-layer schemes and parameters (Frequency-Hopped Binary Phase-Shift Keying). A typical configuration includes a $500 \mathrm{bit} / \mathrm{s}$ command link running along with a 2-10 kbit/s data link, a $27 \mathrm{kHz}$ carrier frequency, $18 \mathrm{kHz}$ of bandwidth, error-correction codes and a communication range of 2-3 km. All parameters are dynamically customizable.

Physical-layer reprogramming has been showcased in [50] (for OFDM), making the Subnero modem the first commercial modem that is natively reprogrammable at all layers of the OSI stack. However, it requires the programming of a TigerSHARC DSP and of a Stratix III FPGA. Alternatively, the UNetStack baseband service makes it possible to reprogram the modem's PHY using the Java/Groovy language. This is achievable as long as the resources of the programmable logic allow the user to employ the FPGA/DSP as a general-purpose processor. For the most computationally efficient implementation, the direct programming of the FPGA/DSP would still be required. UnetStack makes a simulation mode available to test the developed software and agents. The same software can be adapted to work on UnetStack-compatible hardware modems. In September 2013, EvoLogics announced that their WiSE modem series was upgraded to also support UnetStack, in collaboration with Subnero [51].

4) Teledyne Benthos' steps towards reconfigurability (USA, 2013-): Teledyne Benthos has been alleviating some constraints that made their modem closed to external modifications and reprogrammable only by specialized personnel. With reference to the SM-(9)75 modem, a networking API can now be provided to bypass the modem's native networking functions and command the data-link and physical layers (which remain unchanged) through a Modem Management Protocol (MMP) [52], [53]. Based on this flexibility, the University at Buffalo, NY, has been working on a reconfigurable underwater acoustic testbed, where the nodes mount an SM-(9)75 system, complemented by an auxiliary data processor and a Gumstix running control software [52], [54]. The SM-(9)75 modem natively supports the transmission of custom 
waveforms.

\section{European collaborations}

In parallel with the (mainly) USA academic efforts (Sections II-A and II-B), a series of European projects on underwater (network) communications have been running, some of them financed by the national Ministries of Defense of the participating nations (Section II-D1, Section II-D3), and others financed by the European Commission (Brussels) (Section II-D2, Section II-D4).

1) EUROPA MoU UCAC (2005-2008): In a defense cooperation between Sweden, Norway, Finland, Denmark, The Netherlands, Germany and Italy, under the EUROPA Memorandum of Understanding (MoU) [55], covert point-to-point underwater acoustic communications was investigated. In this project, called Unmanned underwater vehicle (UUV) Covert Acoustic Communications (UCAC) [56], successful reception at very low signal-to-noise ratios (down to $-18 \mathrm{~dB}$ ) was achieved using a very robust multiband OFDM modulation [57]. This modulation was developed in Matlab by TNO and Delft University of Technology [58] and included as Matlab-compiled C libraries in the modem software by Patria. The implementation ran in real time under Linux on the Intel Pentium-M processor $(2 \mathrm{GHz})$ of the UCAC modems built by Teledyne RESON (transducer, casing) and Patria (hydrophone, electronics). Transmissions were made between a Kongsberg HUGIN 1000 AUV and its mothership in a 1.5-5 kHz band over ranges up to $20 \mathrm{~km}$ at data rates of $4-80 \mathrm{bit} / \mathrm{s}$. The maximum achieved communication range during early sea tests was $52 \mathrm{~km}[58]$.

2) EU-FP7 UAN (2008-2011) and EU-FP7 CLAM (2011-2013): Publicly funded European research efforts explicitly targeting reconfigurable and reprogrammable underwater acoustic modems started in 2008 with the Underwater Acoustic Networks (UAN, 2008-2011) project under the 7th Framework Programme (FP7) of the European Union. In UAN, a multi-lingual modem was created on which one of three physical-layer protocols could be selected via a specific offline command (no online/in-mission adaptability). Later on, in the EU-FP7 CLAM project 
(CoLlAborative eMbedded networks for submarine surveillance, 2011-2013), a reprogrammable network layer was added to these modems. The modems were cNODE systems of Kongsberg Maritime, interfaced with a Gumstix [59] platform running the SUNSET framework [43].

3) EDA RACUN (2010-2014): In 2010, the UCAC consortium (Section II-D1), minus Finland and Denmark, extended their activities to the other OSI layers in the European Defence Agency (EDA) project RACUN (Robust Acoustic Communications in Underwater Networks, 2010-2014, [60]), in which an ad-hoc deployable underwater acoustic network was successfully demonstrated for relevant military scenarios in shallow waters (mine hunting, maritime surveillance) [61].

In the context of the EDA-RACUN project, two different modems were employed. Develogic's modem (Section II-C1) featured a reprogrammable TI TMS320C6713 300 MHz 32 bit DSP that could host the signal processing required by physical-layer algorithms. The platform included 128 MB SDRAM and TI subsystems for low-power management and signal conditioning, and was quite compact yet reprogrammable at the physical layer. In RACUN, network-layer reprogrammability for the Develogic modem was provided via a serial connection to an external Gumstix [59] or NetDCU [62] platform. In parallel, Saab worked on a research platform supporting both physical- and network-layer reprogrammability. The platform is based on the Open Publish-Subscribe (OPS) architecture [63], which offers asynchronous, efficient and typesecure publish-subscribe operations between processes running on different platforms written in multiple programming languages. During the RACUN sea trials in 2013 and 2014, the Saab modem framework, named SMUM, was running on a high-performance mini-PC (Avalue EPSQM67: Intel i7, 2.1 GHz, 8 GB RAM, 160 GB drive, Windows 7 Pro) resulting in quite bulky nodes, comparable in size to the Scripps system (Section II-B3). For the future, the development of a more compact version is foreseen. Two options were implemented for reconfiguring both the Develogic modem and the Saab modem at the network layer, namely a custom C-based framework and the DESERT Underwater framework [42], [64].

4) EU-FP7 SUNRISE (2013-2016) and University of Twente's Proteus-II: The EU FP7 SUNRISE project [65] has the objective to build a consolidated set of underwater networking 
testbeds [66] and explicitly targets the development of an SDOAM. Among the SUNRISE partners, the NATO STO CMRE has been advocating the design of Software-Defined OpenArchitecture Modems [67]-[69]. The conceptual architecture of CMRE's SDOAM involves multiple protocols at all layers of the OSI stack, whose selection is administered by a policy engine. In turn, this is a starting point to evolve towards cognitive architectures.

The NEMO architecture [70] makes progress towards a concrete implementation of the CMRE SDOAM concept. NEMO is a collection of modules (providing network protocols and functions), services (PHY switching, synchronization, navigation, etc.) drivers, and NATO-specific information bundling such as the codex system [71], tied together by the inter-process communication functionalities of the MOOS middleware [72]. The latest reported development was tested during the REP'14 cruise in July 2014 and included: relative clock synchronization; an information dispatcher, named "herald," that handles the spread of information in the network with a tunable level of accuracy; the codex for information representation; flooding-based routing with duplicate suppression; and a packet-agnostic queuing mechanism.

The Proteus-II modem [73] of the University of Twente (The Netherlands) has been developed in the context of SUNRISE. The Proteus-II inherits and improves over the previous design of the SeaSTAR modem [74]. The modem is designed for low power consumption and is based on two ARM Cortex M4 microcontrollers, one to run the transmitter- and receiver-side processing chains of the acoustic modem (implementing 10- to 200-baud FSK as software radio components) and a second one to run network protocols and perform sensor data processing, including automatic time synchronization functionalities. The modem also exposes an antenna connector that can be used to turn the unit into a radio/acoustic gateway. The power consumption of the modem is sufficiently limited to allow unattended operations for about 1 month using a 5 Ah battery [73]. Buffalo's testbed (Section II-C4) is also part of the SUNRISE project. 


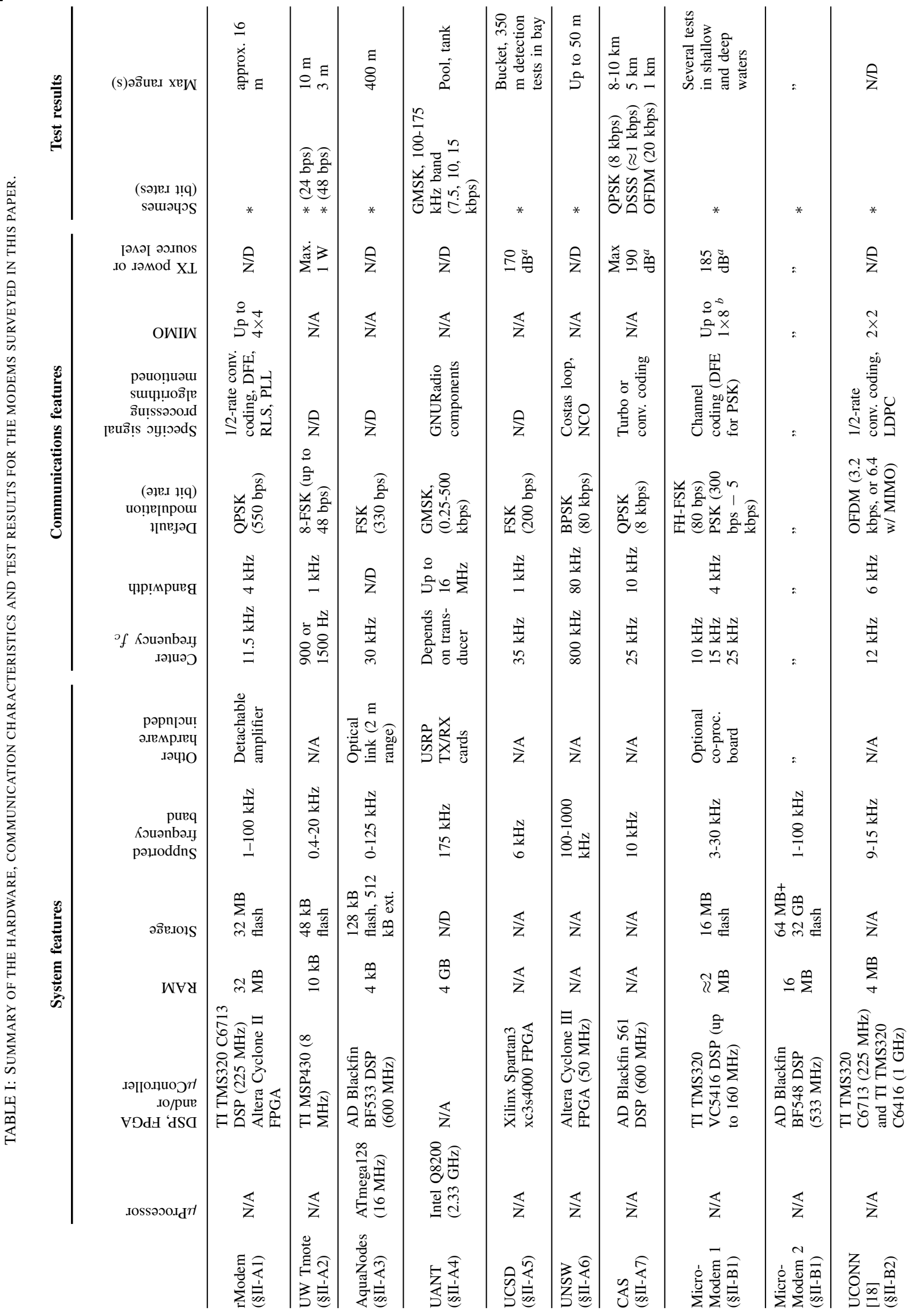




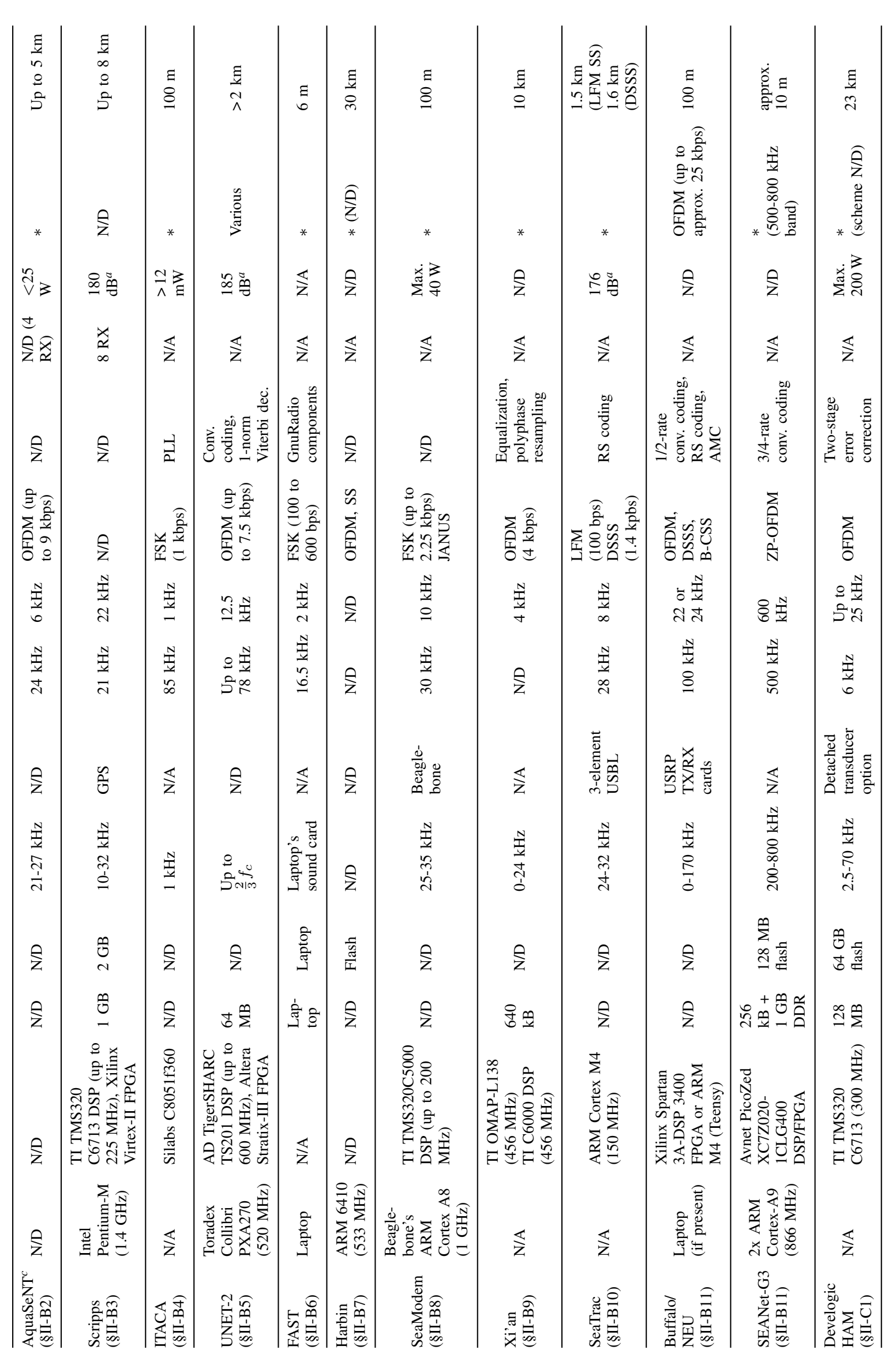




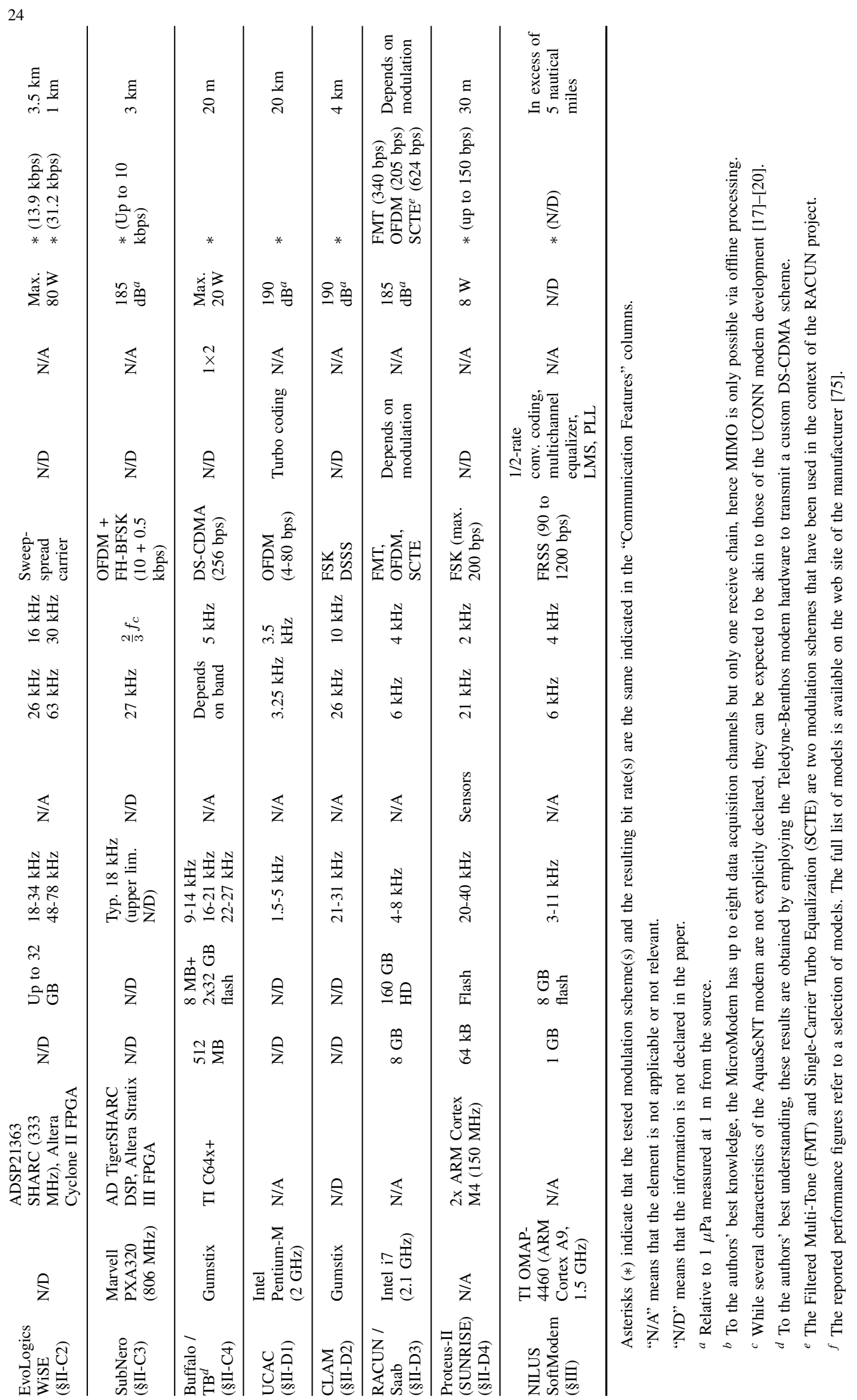


TABLE II

SUMMARY OF THE RECONFIGURABLE, REPROGRAMMABLE AND SOFTWARE-DEFINED MODEMS SURVEYED IN THIS PAPER.

The MEANING OF THE COLUMNS AND BOX COLORS ARE EXPLAINED IN SECTION II-E.

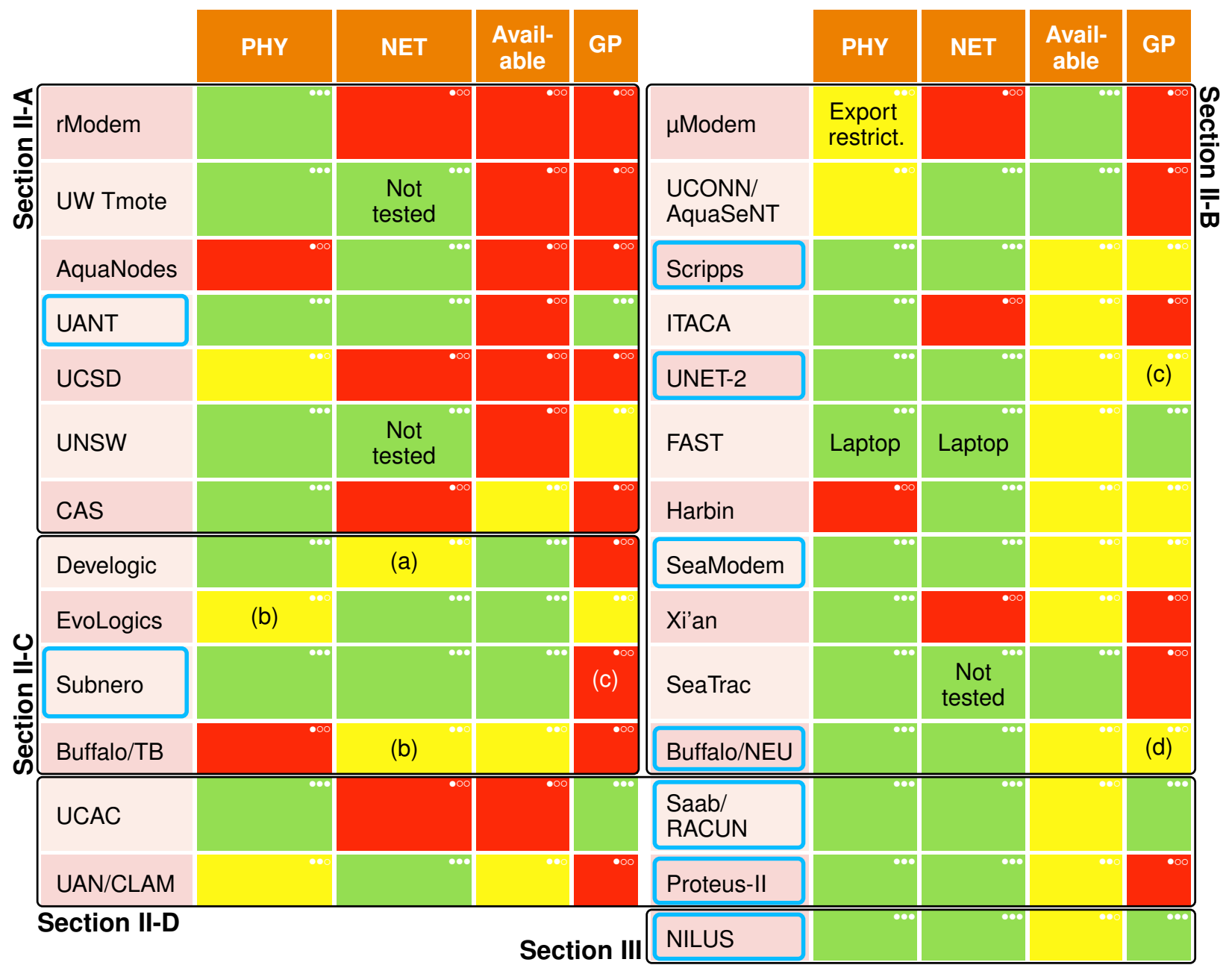

Full/empty circles facilitate reading in a black-and-wide print (one full circle for red, two for yellow and three for green).

(a) The NET protocols attached to the Develogic modem were made reconfigurable in the context of the RACUN project.

(b) To the best of the authors' knowledge, the PHY layer of the EvoLogics modem and the NET layer of the Teledyne modem were reprogrammed in the context of the SUNRISE project.

(c) The UNET-2/Subnero also offer the option to employ the FPGA/DSP as if they were general purpose-processors, and to reprogram them using Java/Groovy.

(d) The Buffalo/NEU entry summarizes the three generations of the SEANet reconfigurable modem prototype: versions 1 and 2 [35], [36], respectively, incorporate an FPGA-based USRP and an ARM Cortex M4-based microcontroller, whereas the latest version [38] is built around a Zynq System-on-Chip architecture with 2x ARM Cortex A9 + FPGA. 


\section{E. Summary}

For the reader's convenience, we summarize the characteristics of the discussed modems in Table I. In addition, Table II classifies the modems by coding the level of reprogrammability (of PHY and NET), the availability, and the generality of the architecture (GP) of each modem in different colors. In Table II, a green PHY box means that the modem's physical layer is reprogrammable by the end-user, a red box means that it is not, while a yellow box means that the user is allowed to reconfigure some PHY parameters, albeit not to completely reprogram the PHY algorithms. The NET boxes indicate the same for the network protocol stack. Availability can be off-the-shelf (green), none (red) or only under the cap of a joint cooperation (yellow). In the context of such a cooperation, it may also be possible to reprogram PHY schemes or NET protocols that would normally be only reconfigurable or closed. Finally, the programmable platform can be a general-purpose (GP) processor (green), a dedicated processor such as a DSP or microcontroller (red), or both (yellow). To facilitate reading in a black-and-white print, we provided three full/empty circles in the top-right corner of each table cell, where one full circle represents "red," two represent "yellow," and three represent "green." The GP column gives an idea of the accessibility and flexibility of a modem to the user. In general, a thorough knowledge of the specific hardware and/or software architecture is needed to modify a modem implementation on a dedicated architecture, such as a DSP or microcontroller. On the contrary, specialized knowledge is less needed when the modem code is written using standard languages (e.g., $\mathrm{C} / \mathrm{C}++$ ) and runs under an operating system on a general-purpose processor. The blue frames highlight doubly-reprogrammable standalone underwater acoustic modems. Both Table I and Table II also include the NILUS SoftModem, which is presented and evaluated in the following section.

\section{The NILUS SoftModeM}

The NILUS bottom node is a lightweight underwater sensor node developed by the Norwegian Defence Research Establishment (FFI) [76]. Extensive experience with this system has been 
gained in the NATO Joint Research Project NGAS (Next Generation Autonomous Systems), the EDA project RACUN [60], and in national Norwegian trials. In the framework of a bilateral Netherlands-Norway defense research cooperation (2014-), a new version of the NILUS node (MK 2) was developed, having an improved sensor suite and more processing capabilities. Also, the Teledyne Benthos modems of the original NILUS (MK 1) nodes are (optionally) replaced by a software-defined modem developed at TNO, which is presented below. The modem has been designed to be able to run on general-purpose processors only, and can thus be fully reprogrammed using well-known programming languages acting on well defined interfaces. In the same vein, the modem architecture is devoid of DSPs and FPGAs, which require specialized programming efforts and tools. The NILUS MK 2 differentiates itself from most currently available reprogrammable and reconfigurable modems, which often rely on specialized hardware to carry out PHY operations (see Table II). In this respect, the NILUS MK 2 makes significant steps towards the realization of a Software-Defined Open Architecture Modem (SDOAM).

\section{A. Modem hardware}

The modem's transducer is mounted on a subsurface buoy (see Fig. 1) hovering above the NILUS frame, about $6 \mathrm{~m}$ above the sea floor. The buoy includes the amplifier, the impedancematching network, and the projector $(3-11 \mathrm{kHz},-3 \mathrm{~dB}$ bandwidth). The receiving hydrophone and pre-amplifier are mounted on the tripod structure, approximately $1 \mathrm{~m}$ above the sea floor. This hydrophone is the top element of a tetrahedral array: the other hydrophone elements are only used for the sensor capabilities of the NILUS node for the time being, but are potentially also available for modem reception.

The NILUS SoftModem framework, consisting of the physical (PHY), media-access control (MAC) and network (NET) layers, as well as audio and data interfaces, is running under GNU/Linux on the Variscite System-on-Module board VAR-SOM-OM44, which is based on

a Texas Instruments OMAP4460 1.5 GHz Dual-Core ARM Cortex-A9 processor [77]. This compact and energy-efficient yet powerful computational platform, developed for the smart- 


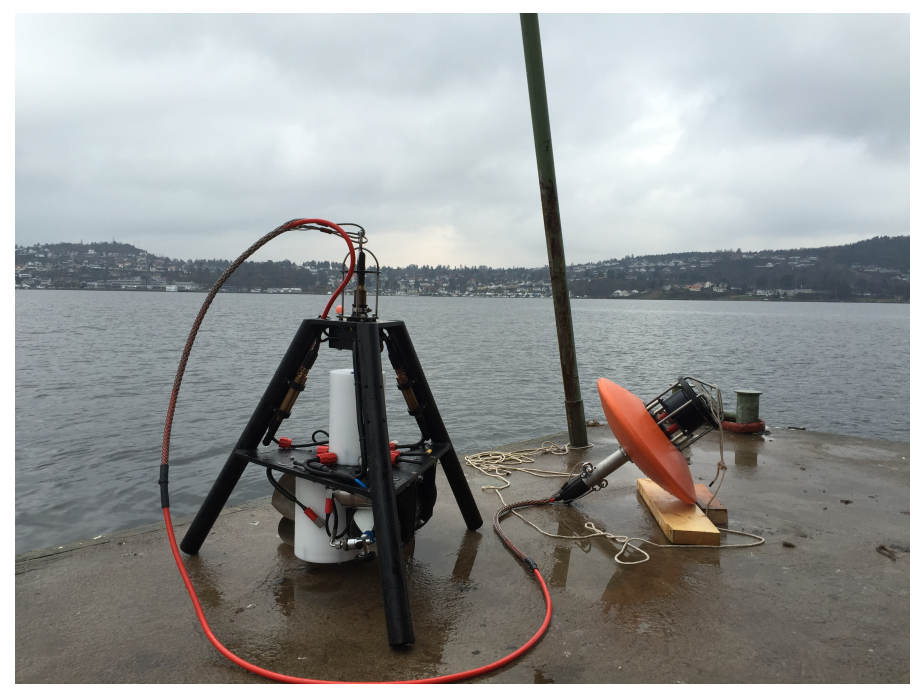

Fig. 1. The NILUS MK 2 bottom node, with the new software-defined modem (transducer in special float shape for stability in currents).

phone/tablet industry, has sufficient resources (7500 MIPS, 1 GB RAM, 8 GB MicroSD) for running a state-of-the-art communications stack programmed in an architecture-independent highlevel programming language (ISO C, C++), while still being compact and economic in terms of cost and power usage, and substantially easier to program than DSP boards. The VAR-SOMOM44 is equipped with a dedicated high-quality audio codec, the TWL6040 [78].

\section{B. Modem software}

Fig. 2 shows the architecture and data-flow diagram of the NILUS SoftModem, where the "NILUS Modem Software" block contains the modulation and demodulation routines (PHY), which interfaces with the "MAC and Network layers" via standard TCP connections. The connection between the PHY block and the "Transducer and Preamp," including buffering, makes use of the Advanced Linux Sound Architecture (ALSA) driver for the integrated sound card. The data connection between the NET layer and the "NILUS Software" (APP layer, on a separate processing board) is realized via a UART interface. The data from the receiving hydrophone preamp comes in by the "Common System Bus." The hydrophone data is published on this 


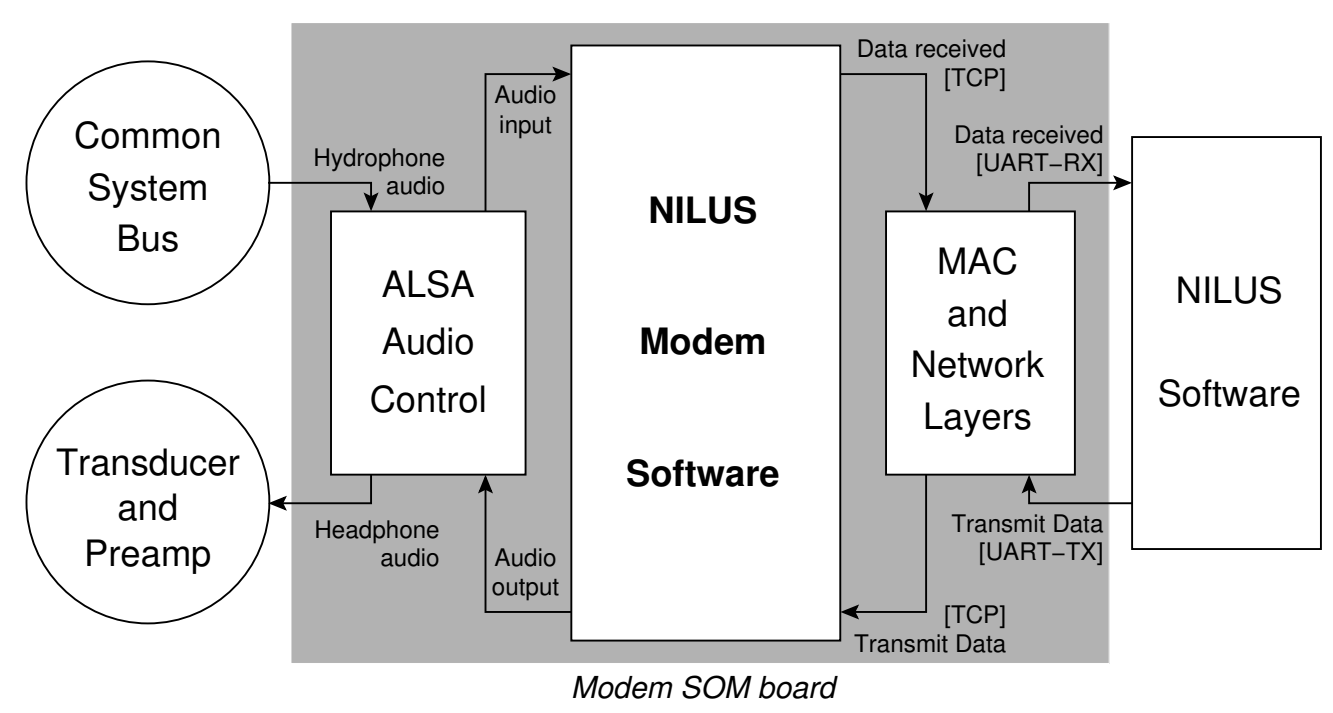

Fig. 2. Architecture and data-flow diagram of the NILUS SoftModem [79].

digital bus in an ALSA-compatible PCM format. The "ALSA Audio Control" can therefore rely on the proper ALSA driver for retrieving the hydrophone data.

The modulation scheme employed on the NILUS MK 2 node involves modulating root-raised cosine-windowed QPSK pulses, which are spread through the Frequency-Repetition SpreadSpectrum (FRSS) scheme [80], a coherent multiband spread-spectrum method based on [81]. Fig. 3 shows an example of an FRSS signal carrying 256 bits at a bit rate of approximately 90 bit/s, having a length of approximately $2.9 \mathrm{~s}$. The scheme was originally developed and extensively tested in Matlab at TNO and FFI. The method applies a linear (decision feed-forward) multichannel equalizer with an ultra-wideband phase-locked-loop solution to several "frequency-band channels" containing the same information but multiplied by a spreading code across frequency bands. The filter taps are updated by the LMS algorithm, and a Viterbi algorithm is used to decode the rate-1/2 convolutional coding. Using the output SNR [82], the bit probabilities are computed, which are used as "soft information" for the Viterbi decoder. The FRSS modulation is programmed in $\mathrm{C}++11$ in a modular Qt framework. Public GPL-licensed libraries are employed for the FFT (FFTW), linear matrix algebra operations (Armadillo) and resampling routines (Secret Rabbit Code). 


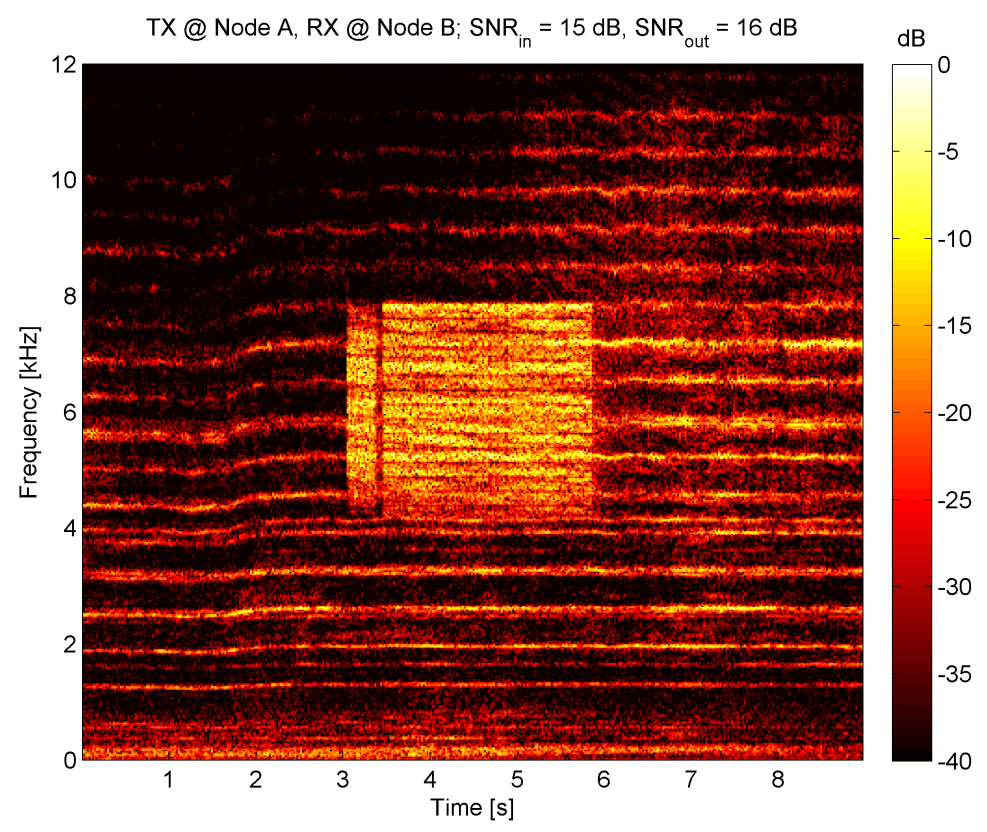

Fig. 3. Example FRSS signal (approx. 2.9 s, 256 info bits, approx. 90 bit/s) in the presence of nearby ship noise. The message was correctly decoded at node B of Fig. 4(a).

For the MAC protocol, ALOHA with carrier sensing (CS) is employed, while a restricted flooding network protocol named Dflood, developed by FFI in the context of the RACUN project (see Section II-D3), is employed at the network layer [83], [84]. The APP layer expresses commands and information reports of different types using standard packets of size equal to 128 bits. The entire network protocol stack including MAC and Dflood runs under the ns2-based DESERT Underwater framework [41], [42], with ns2 running in parallel with the modem (PHY) on the SoftModem processor board. By virtue of this, it is possible to use exactly the same software for the network protocol stack in network simulations and at sea.

\section{Experimental results}

The first open-water tests for the new NILUS nodes were successfully performed in November 2014 in the inner harbor of Horten (Indre Havn), Norway [82]. The applied frequency band is the RACUN band, from 4 to $8 \mathrm{kHz}$, which corresponds to the $-1 \mathrm{~dB}$ bandwidth of the modem's 
transducer. In a 4-node network with an average internode distance of about $1 \mathrm{~km}$, an overall packet delivery ratio (PDR) at the physical layer of approximately 0.86 was achieved. This physical-layer PDR was defined as the ratio between the number of messages that were received error-free and the number of detected messages. Given the initial difficulties of a first trial, the use of a relatively low source level (SL), and the presence of a motorboat sailing through the network, this PDR was found to be satisfactory. This judgement was confirmed by the next trial in March 2015 in the shallow Haringvliet estuary in The Netherlands, where a (PHY) PDR of about 0.95 was achieved in a 4-node network with an average internode distance of about $3 \mathrm{~km}$ (at a higher SL). Both trials featured direct line of sight between all nodes.

After these initial trials, a project demonstration trial was performed in April 2015, again in the inner harbor of Horten and this time extended to deeper waters in the Oslofjord. One of the scenarios tested was a relay connection between the harbor and the fjord, see Fig. 4(a). This experiment was conducted in the morning of April 16, in nice weather conditions (clear sky, some wind, sea state approximately 1). The nodes were deployed at bottom depths of approximately $8 \mathrm{~m}$ (position C), $18 \mathrm{~m}$ (positions A, B and D) and $80 \mathrm{~m}$ (positions E, F and G). Diverse bottom sediments were present in the trial area (mud, sand, rocks). The properties of the water column were sampled using a CTD probe. Not much temporal variability was observed in the collected sound-speed profiles. The mainly upward-refracting sound-speed profile resulted in a strong influence of the sea-surface scattering on the time delay (multipath, reverberation) and Doppler spread. Furthermore, there was some ship traffic, especially in the Oslofjord.

The (PHY) PDR statistics in the multihop network were collected for more than an hour, resulting in the network connectivity figures displayed in Fig. 4(b). The number of packets received by each node during this time varied between 7 and 29, providing a total of 273 investigated packet receptions in the network. The overall PDR was $218 / 273 \approx 0.80$ for internode distances from about $450 \mathrm{~m}$ to $2,350 \mathrm{~m}$ (average about $1210 \mathrm{~m}$ ). The results show that the network featured long-range point-to-point connections in deep water with direct line of sight (e.g., $F \leftrightarrow G$ ), whereas hopping was needed for large ranges in shallow water without direct line 
of sight (e.g., $\mathrm{C} \leftrightarrow \mathrm{F}$, up to 3 hops). Without relay nodes $\mathrm{C}$ and $\mathrm{D}$, the nodes in the harbor would have been disconnected from the nodes in the fjord. In fact, as node $\mathrm{C}$ was originally not present in the network, nodes B and D were initially disconnected. After about 40 minutes, the node at position A (base station at barge) was moved to position $\mathrm{C}$ (base station at support vessel), thus connecting the fjord with the inner harbor. The PDR statistics for both time intervals have been merged in the connectivity graph. On the network-layer level, the PDR was 1.0 between all NILUS nodes and the base station, achieved through the retransmission scheme of the applied NET protocol (i.e., no unique packets were lost after max. 1 retransmission).

It is interesting to observe that perfect links $(\mathrm{F} \leftrightarrow \mathrm{G}, \mathrm{E} \leftrightarrow \mathrm{F})$ co-exist close to paths with (almost) no connection at all $(\mathrm{E} \leftrightarrow \mathrm{G})$. This extreme difference is believed to be mainly due to the presence of an underwater hill between positions E and G. At these positions, the local depth is about $80 \mathrm{~m}$, while the hill rises up to a broad plateau of about $12 \mathrm{~m}$ depth around the line E-G. With the transmitter being approximately $6 \mathrm{~m}$ above the bottom and the receiver approximately $1 \mathrm{~m}$, there is no direct line of sight between the nodes at $\mathrm{E}$ and $\mathrm{G}$ and all communication between $\mathrm{E}$ and $\mathrm{G}$ goes via surface-reflected paths. On the other hand, there is a direct line of sight between $\mathrm{E}$ and $\mathrm{F}$. For the line F-G, an offshoot of the hill just seems to prevent a direct line of sight, but still this link was working well, most probably because $\mathrm{F}$ is much farther from the hill than E. Messages were received by node $\mathrm{G}$ from nodes $\mathrm{D}, \mathrm{E}$ and $\mathrm{F}$ in the same time frame, so the background noise level was the same for all receptions. Also, signal collisions were equally spread between messages from all three nodes. The fact that the longest direct link $(\mathrm{F} \leftrightarrow \mathrm{G})$ was perfect suggests that the modem source level was more than sufficient and that the point-topoint communication range was limited by acoustic shadowing and/or delay-Doppler spreading, especially in the very shallow sections of the network area. This nicely illustrates the usefulness of multihop networks with alternative routes to the destination.

The successful demonstration of the NILUS MK 2 bottom nodes, including the NILUS SoftModems, show that generic (open) architectures can be applied to underwater acoustic modems, while still meeting (semi) operational requirements in terms of processing power, size 


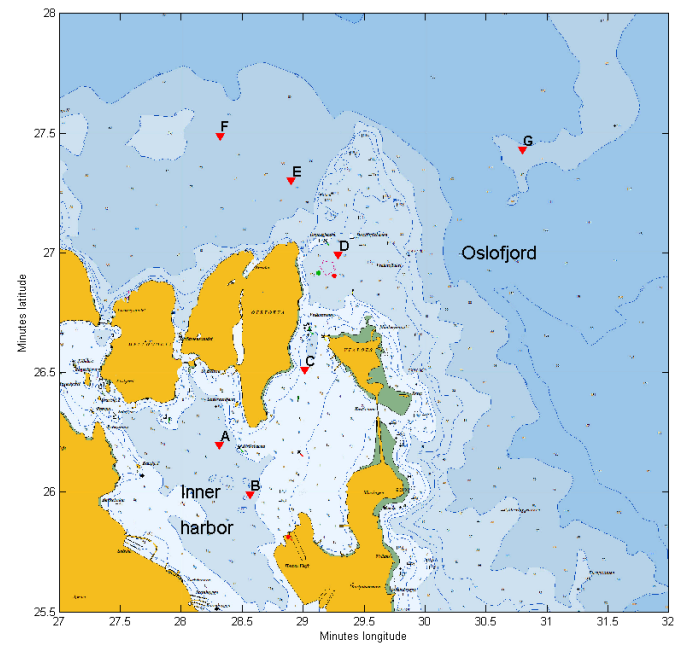

(a)
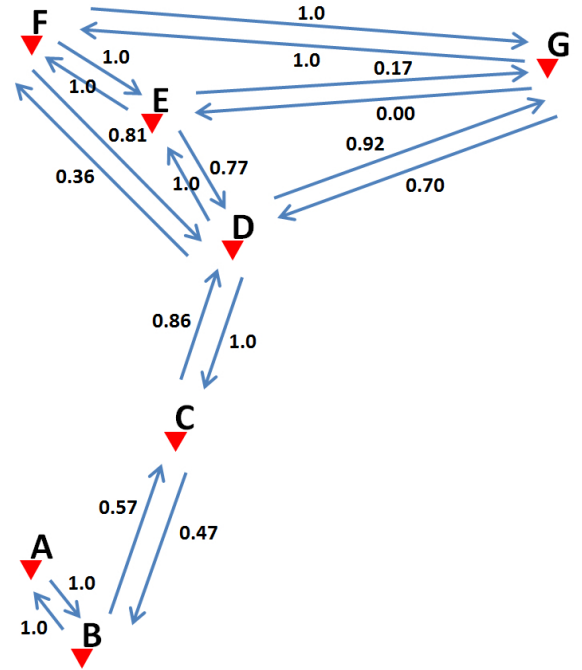

(b)

Fig. 4. (a) Map of Horten harbor and Oslofjord (mosaic of TELchart/C-Map screenshots) with node positions indicated (in minutes relative to $59^{\circ} \mathrm{N}$ and $10^{\circ} \mathrm{E}$ ). (b) Physical-layer packet delivery ratios in the experimental deployment on April 16,2015 .

and endurance. The processing power should be sufficient for real-time execution of a "heavyduty" network stack (robust, high throughput), the size should be small enough to fit into "manportable" sensor systems [76], while the required endurance depends on the application and operational scenario.

\section{Conclusions}

Our historical review showed that the most flexible reprogrammable underwater acoustic modem is a software-defined modem, where specific tools make it possible for the user to apply changes to (part of) the modem firmware without involving the modem manufacturer. For some modems, reprogrammability applies only to the networking components, for other modems only to the physical layer, and in several cases, only some level of parameter reconfiguration is allowed to the user. Notably, in seven cases presented in the review (UANT, Scripps, UNET-2, SeaModem, Buffalo/NEU, Subnero and Saab) both the physical and the network layers are fully reprogrammable by the end user. These doubly-reprogrammable modems are marked in Table II with blue frames, together with the NILUS SoftModem. Open-architecture modems, featuring 
a fully transparent and free hardware/software architecture, are still missing for the underwater community, but they are advocated by several research efforts at different institutions, and further advances are expected in the coming years.

Notably, a number of the reprogrammable modems reviewed in this paper (AquaNodes, UCONN, Scripps, SeaModem, SeaTrac, Buffalo/NEU, Develogic, EvoLogics, UNET-2/Subnero, UCAC, UAN/CLAM, Saab) have actually seen seaborne tests and a development phase in shallow-water environments, while other reprogrammable modems were at an earlier stage of development (e.g., detached electronics, in-tank transducer, offline tests). Some modems also demonstrated MIMO functionalities.

Whereas most reviewed modems are research prototypes that are not commercially available, several COTS modems implement software-defined functions. Good examples are Develogic, who opened their physical layer, EvoLogics, who have implemented a flexible network layer, and Subnero, who made it possible to customize both the physical layer and the network protocols.

In addition to the review of these activities, we presented the specific setup of the NILUS SoftModem, jointly designed by TNO and FFI. This modem is based on powerful generalpurpose-architecture computation platforms, such as the Dual-Core ARM Cortex-A9 board, which enables the real-time execution of both physical-layer and network-layer protocols programmed in generic high-level languages. Therefore, the PHY/NET programmer does not have to worry about the hardware details, since one only has to comply with well-defined APIs in a highlevel programming framework. To the best of the authors' knowledge, the NILUS SoftModem is the only one among all surveyed modems showing this level of flexibility, along with a compact rugged design and (semi) operational readiness. In some cases (notably, Develogic [39], UNETII [26] and SubNero [47]), the system is arranged so that high-level programming languages can be employed to directly reprogram the DSP/FPGA that run PHY algorithms on the modem. This is possible as long as the DSP/FPGA memories are large enough, but it will not result in the most efficient implementation. These considerations are overcome by the design of the NILUS SoftModem, which is fully based on general-purpose computing architectures. This compact and 
efficient general-purpose platform, a product of smartphone industry, may provide the bridge to software-defined open-architecture underwater acoustic modems becoming common practice.

We remark that the flexibility of a softmodem running under an operating system on a generalpurpose processor comes at a price, such as increased power usage and wake-up time. This is a trade-off that has to be made by the end-user and will depend on the use case, on the scenario and on flexibility requirements. However, as long as there are no heavy-duty underwater communication stacks being standardized and implemented on all COTS underwater modems, a softmodem may be the only way to achieve interoperability in a heterogeneous network, e.g., as set up by allied navies in a joint operation. Our vision for the future is to further exploit the NILUS SoftModem's flexibility by developing a smart adaptive communication stack, i.e., a modem that can switch autonomously between protocols, frequency bands, data rates, message lengths, etc., based on environmental conditions and operational needs.

\section{ACKNOWLEDGMENTS}

The review in Section II has been partly performed in the framework of the EDA project RACUN, funded by the ministries of defense of Germany, Italy, Netherlands, Norway and Sweden. The work in Section III has been performed in the context of a bilateral NetherlandsNorway defense cooperation between the Defence Materiel Organisation (DMO, NL) and the Norwegian Defence Research Establishment (FFI, NO). The work of Paolo Casari has also been

partially supported by the EU, FP7 project AMAROUT II (G.A. no. 291803). A preliminary version of this paper was presented at the UCOMMS 2014 conference, Sestri Levante, Italy, September 2014.

The authors would like to thank the NILUS SoftModem team at TNO and FFI, especially Mathieu Colin (TNO), Paul van Walree (FFI), and Joachim Eastwood (FFI).

\section{REFERENCES}

[1] E. Jones, "The application of software radio techniques to underwater acoustic communications," in Proc. IEEE/OES Oceans, Aberdeen, Scotland, Jun. 2007. 
[2] E. M. Sözer and M. Stojanovic, "Reconfigurable acoustic modem for underwater sensor networks," in Proc. ACM WUWNet, Los Angeles, CA, Sep. 2006.

[3] L. Freitag, M. Grund, S. Singh, J. Partan, P. Koski, and K. Ball, “The WHOI Micro-Modem: An acoustic communications and navigation system for multiple platforms," in Proc. MTS/IEEE Oceans, Washington DC, USA, 2005.

[4] M. Aydinlik, A. T. Ozdemir, and M. Stojanovic, "A physical layer implementation on reconfigurable underwater acoustic modem,” in Proc. MTS/IEEE Oceans, Québec City, Canada, Sep. 2008.

[5] R. Jurdak, C. V. Lopes, and P. Baldi, "Software acoustic modems for short range mote-based underwater sensor networks," in Proc. IEEE Oceans, Singapore, May 2006.

[6] R. Jurdak, P. Aguiar, P. Baldi, and C. V. Lopes, "Software modems for underwater sensor networks," in Proc. IEEE/OES Oceans, Aberdeen, Scotland, Jun. 2007.

[7] I. Vasilescu, C. Detweiler, and D. Rus, “AquaNodes: An underwater sensor network," in Proc. ACM WUWNet, Montréal, Canada, Sep. 2007.

[8] D. Torres, J. Friedman, T. Schmid, and M. B. Srivastava, "Software-defined underwater acoustic networking platform," in Proc. ACM WUWNet, Berkeley, CA, Nov. 2009.

[9] D. Torres, J. Friedman, T. Schmid, M. B. Srivastava, Y. Noh, and M. Gerla, "Software-defined underwater acoustic networking platform and its applications," Elsevier Ad Hoc Networks S.I. “Advances in underwater communications and networks", vol. 34, pp. 252-264, 2015.

[10] B. Benson, Y. Li, B. Faunce, K. Domond, D. Kimball, C. Schurgers, and R. Kastner, "Design of a low-cost underwater acoustic modem,” IEEE Embedded Syst. Lett., vol. 2, no. 3, pp. 58-61, Sep. 2010.

[11] N. Nowsheen, C. Benson, and M. Frater, "Design of a high frequency FPGA acoustic modem for underwater communication,” in Proc. MTS/IEEE OCEANS, Sydney, Australia, May 2010.

[12] — "A high data-rate, software-defined underwater acoustic modem," in Proc. MTS/IEEE OCEANS, Seattle, WA, Sep. 2010.

[13] C. Benson, M. Ryan, and M. Frater, "Towards robust high data-rate hydro-acoustic modems," in Proc. MTS/IEEE OCEANS, Hampton Roads, VA, Oct. 2012.

[14] Y. Li and H. Huang, "The design and experiment of a software-defined acoustic modem for underwater sensor network," in Proc. MTS/IEEE OCEANS, Sydney, Australia, May 2010.

[15] E. Gallimore, J. Partan, I. Vaughn, S. Singh, J. Shusta, and L. Freitag, "The WHOI Micromodem-2: A scalable system for acoustic communications and networking," in Proc. MTS/IEEE Oceans, Seattle, WA, USA, 2010.

[16] Woods Hole Oceanographic Institution, Acoustic Communications Group, “Micromodem pages," accessed: June 2016. [Online]. Available: http://acomms.whoi.edu/micro-modem/

[17] B. Li, J. Huang, S. Zhou, K. Ball, M. Stojanovic, L. Freeitag, and P. Willett, "MIMO-OFDM for high-rate underwater acoustic communications," IEEE J. Ocean. Eng., vol. 34, no. 4, pp. 634-644, Oct. 2009.

[18] H. Yan, L. Wan, S. Zhou, Z. Shi, J.-H. Cui, J. Huang, and H. Zhou, "DSP based receiver implementation for OFDM acoustic modems," Elsevier Physical Communication, vol. 5, no. 1, pp. 22-32, Mar. 2012. 
[19] S. Zhou and Z. Wang, OFDM for Underwater Acoustic Communications. John Wiley \& Sons, May 2014.

[20] L. Wan, H. Zhou, X. Xu, Y. Huang, S. Zhou, Z. Shi, and J.-H. Cui, "Adaptive modulation and coding for underwater acoustic OFDM," IEEE J. Ocean. Eng., vol. 40, no. 2, pp. 327-336, Apr. 2015.

[21] S. N. Le, Z. Peng, J.-H. Cui, H. Zhou, and J. Liao, "SeaLinx: A multi-instance protocol stack architecture for underwater networking," in Proc. ACM WUWNet, Kaohsiung, Taiwan, Nov. 2013.

[22] Y. Su, Y. Zhang, S. Le, H. Mo, L. Wei, Y. Huang, Z. Peng, and J.-H. Cui, "A versatile lab testbed for underwater sensor networks," in Proc. MTS/IEEE OCEANS, San Diego, CA, Sep. 2013.

[23] W. S. Hodgkiss and J. D. Skinner, "A multichannel software-defined acoustic modem,” in Proc. ECUA, Istanbul, Turkey, Jul. 2010.

[24] A. Sánchez, S. Blanc, P. Yuste, and J. J. Serrano, "A low cost and high efficient acoustic modem for underwater sensor networks," in Proc. IEEE/OES Oceans, Santander, Spain, Jun. 2011.

[25] A. Sánchez, S. Blanc, P. Yuste, A. Perles, and J. J. Serrano, "An ultra-low power and flexible acoustic modem design to develop energy-efficient underwater sensor networks," MDPI Sensors, vol. 12, no. 6, pp. 6837-6856, 2012. [Online]. Available: http://www.mdpi.com/1424-8220/12/6/6837

[26] M. Chitre, I. Topor and T.-B. Koay, "The UNET-2 modem: An extensible tool for underwater networking research," in Proc. MTS/IEEE OCEANS, Yeosu, South Korea, May 2012.

[27] S. Shahabudeen, M. Chitre, M. Motani, and A. Siah, "Unified simulation and implementation software framework for underwater MAC protocol development," in Proc. MTS/IEEE OCEANS, Biloxi, MS, Oct. 2009.

[28] N. Ahmed, W. bin Abbas, and A. A. Syed, "A low-cost and flexible underwater platform to promote experiments in UWSN research," in Proc. ACM WUWNet, Los Angeles, CA, Nov. 2012.

[29] J. Zhang, G. Qiao, and C. Wang, "An intelligent management platform for underwater acoustic modem," in Proc. ISCID, Hangzhou, China, Oct. 2012.

[30] G. Cario, A. Casavola, M. Lupia, and C. Rosace, "SeaModem: a low-cost underwater acoustic modem for shallow water communication,” in Proc. MTS/IEEE Oceans, Genova, Italy, May 2015.

[31] R. Petroccia, G. Cario, M. Lupia, V. Djapic, and C. Petrioli, "First in-field experiments with a "bilingual" underwater acoustic modem supporting the JANUS standard," in Proc. MTS/IEEE Oceans, Genova, Italy, May 2015.

[32] J. Potter, J. Alves, D. Green, G. Zappa, I. Nissen, and K. McCoy, "The JANUS underwater communications standard,” in Proc. UCOMMS, Sestri Levante, Italy, Sep. 2014.

[33] C. Wang, Q. Zhang, Z. Yan, J. Han, K. Lei, and L. Zhang, "Implementation of underwater acoustic modem based on the OMAP-L138 processor," in Proc. IEEE ICSPCC, Guilin, China, Aug. 2014.

[34] J. A. Neasham, G. Goodfellow, and R. Sharphouse, "Development of the "Seatrac" miniature acoustic modem and USBL positioning units for subsea robotics and diver applications," in Proc. MTS/IEEE Oceans, Genova, Italy, May 2015.

[35] E. Demirors, B. G. Shankar, G. E. Santagati, and T. Melodia, "SEANet: A software-defined acoustic networking framework for reconfigurable underwater networking," in Proc. ACM WUWNet, Washington, DC, Oct. 2015. 
[36] E. Demirors, G. Sklivanitis, T. Melodia, S. Batalama, and D. Pados, "Software-defined underwater acoustic networks: toward a high-rate real-time reconfigurable modem,” IEEE Commun. Mag., vol. 53, no. 11, pp. 64-71, Nov. 2015.

[37] E. Demirors, G. Sklivanitis, G. E. Santagati, T. Melodia, and S. N. Batalama, "Design of a software-defined underwater acoustic modem with real-time physical layer adaptation capabilities," in Proc. ACM WUWNet, Rome, Italy, Nov. 2014.

[38] E. Demirors and T. Melodia, "SEANet G3: High-data-rate software-defined underwater acoustic network platform," in Proc. UCOMMS, Lerici, Italy, Sep. 2016, accepted.

[39] F. Berning, T. Radtke, S. Rautenberg, M. Motz, and I. Nissen, "A realization of the software defined radio concept in an underwater communication modem," in Proc. UCOMMS, Sestri Levante, Italy, Sep. 2014.

[40] EvoLogics, "White line science edition (WiSE) modems," accessed: June 2016. [Online]. Available: http: //www.evologics.de/en/products/developer_tools/index.html

[41] R. Masiero, S. Azad, F. Favaro, M. Petrani, G. Toso, F. Guerra, P. Casari, and M. Zorzi, "DESERT Underwater: an NS-Miracle based framework to DEsign, Simulate, Emulate and Realize Test-beds for Underwater network protocols," in Proc. IEEE/OES Oceans, Yeosu, South Korea, May 2012.

[42] P. Casari, C. Tapparello, I. Calabrese, F. Favaro, G. Toso, S. Azad, R. Masiero, and M. Zorzi, "Open-source suites for the underwater networking community: WOSS and DESERT underwater," IEEE Network, S.I. Open Source for Networking: Development and Experimentation, vol. 28, no. 5, pp. 38-46, Sep. 2014.

[43] C. Petrioli, R. Petroccia, and D. Spaccini, "SUNSET version 2.0: Enhanced framework for simulation, emulation and real-life testing of underwater wireless sensor networks," in Proc. ACM WUWNet, Kaohsiung, Taiwan, Nov. 2013.

[44] O. Kebkal, M. Komar, K. Kebkal, and R. Bannasch, "D-MAC: media access control architecture for underwater acoustic sensor networks," in Proc. IEEE/OES Oceans, Santander, Spain, Jun. 2011.

[45] —-, "Long-baseline hydro-acoustic positioning using D-MAC communication protocol," in Proc. MTS/IEEE Oceans, Yeosu, South Korea, May 2012.

[46] O. Kebkal, V. Kebkal, and K. Kebkal, "EviNS: A framework for development of underwater acoustic sensor networks and positioning systems," in Proc. MTS/IEEE Oceans, Genova, Italy, May 2015.

[47] NUS ARL, Subnero, "UNET - The Underwater NETworks project," accessed: June 2016. [Online]. Available: http://www.unetstack.net

[48] M. Chitre, R. Bhatnagar, and W.-S. Soh, "UnetStack: An agent-based software stack and simulator for underwater networks," in Proc. MTS/IEEE OCEANS, St. John's, Canada, Sep. 2014.

[49] Subnero, “Underwater modem overview," accessed: June 2016. [Online]. Available: http://subnero.com/technology/modem/

[50] M. Chitre, R. Bhatnagar, M. Ignatius, and S. Suman, "Baseband signal processing with UnetStack," in Proc. UCOMMS, Sestri Levante, Italy, Sep. 2014.

[51] “EvoLogics newsletter," accessed: June 2016. [Online]. Available: http://www.evologics.de/en/news.html?newsman_news_ $\mathrm{id}=51$

[52] H. Kulhandjian, L. Kuo, and T. Melodia, "Extended abstract: Development of a reconfigurable underwater networking testbed," in Proc. ACM WUWNet, Los Angeles, CA, Nov. 2012. 
[53] D. Green and S. McManus, "Smart modems - Underwater capabilities beyond communications," in Proc. MTS/IEEE Oceans, Yeosu, South Korea, May 2012.

[54] H. Kulhandjian, L.-C. Kuo, T. Melodia, D. A. Pados, and D. Green, "Towards experimental evaluation of software-defined underwater networked systems," in Proc. UComms, Sestri Levante, Italy, Sep. 2012.

[55] European Defence Agency (EDA), "European Understandings for Research Organisation, Programmes and Activities (EUROPA)," accessed: July 2015. [Online]. Available: http://www.eda.europa.eu/RandTUserGuide/docs/ default-document-library/europa_mou_version_signed_150501.pdf

[56] P. van Walree, T. Ludwig, C. Solberg, E. Sangfelt, A. Laine, G. Bertolotto, and A. Ishøy, "UUV covert acoustic communications," in Proc. UAM, Nafplion, Greece, Jun. 2009.

[57] H. Dol and P. van Walree, "Underwater acoustic communication research at TNO - past and present," in Proc. IEEE/OES Oceans, Santander, Spain, Jun. 2011.

[58] G. Leus and P. A. van Walree, "Multiband OFDM for covert acoustic communications," IEEE J. Sel. Areas Commun., vol. 26, no. 9, pp. 1662-1673, Dec. 2008.

[59] “Gumstix home page,” accessed: June 2016. [Online]. Available: http://www.gumstix.com/

[60] J. Kalwa, "The RACUN project: Robust acoustic communications in underwater networks - an overview," in Proc. IEEE/OES Oceans, Santander, Spain, Jun. 2011.

[61] P. Casari, J. Kalwa, M. Zorzi, S. Nasta, S. Schreiber, R. Otnes, P. van Walree, M. Goetz, A. Komulainen, B. Nilsson, J. Nilsson, T. Øberg, I. Nissen, H. Strandberg, H. Dol, G. Leus, and F. Pacini, “Ad hoc acoustic networks of heterogeneous nodes for tactical underwater missions," 2016, [submitted].

[62] F\&S Elektronik Systeme GmbH, "NetDCU platform,” accessed: June 2016. [Online]. Available: http://www.fs-net.de/en/ products/netdcu/

[63] A. Gravenstam, “OPS Open Publish-Subscribe middleware," accessed: June 2016. [Online]. Available: http: //code.google.com/p/ops/

[64] C. Tapparello, P. Casari, G. Toso, I. Calabrese, R. Otnes, P. van Walree, M. Goetz, I. Nissen, and M. Zorzi, "Performance evaluation of forwarding protocols for the RACUN network," in Proc. ACM WUWNet, Kaohsiung, Taiwan, Nov. 2013.

[65] “SUNRISE FP7 project web site," accessed: June 2016. [Online]. Available: http://fp7-sunrise.eu/

[66] C. Petrioli, R. Petroccia, D. Spaccini, A. Vitaletti, T. Arzilli, D. Lamanna, and A. Galizia, “The SUNRISE gate: Accessing the SUNRISE federation of underwater test-beds," in Proc. UCOMMS, Sestri Levante, Italy, Sep. 2014.

[67] J. Potter, A. Berni, J. Alves, D. Merani, G. Zappa, and R. Been, "Underwater communications protocols and architecture developments at NURC," in Proc. IEEE/OES Oceans, Santander, Spain, Jun. 2011.

[68] J. Potter, J. Alves, A. Vermeij, T. Furfaro, N. Jourden, G. Zappa, A. Berni, and D. Merani, "Software-defined open architecture modem development at CMRE,” NATO STO CMRE, Tech. Rep. CMRE-FR-2013-023, 2013.

[69] J. Potter, J. Alves, T. Furfaro, A. Vermeij, N. Jourden, G. Zappa, A. Berni, and D. Merani, "Software defined open architecture modem development at CMRE,” in Proc. UCOMMS, Sestri Levante, Italy, Sep. 2014. 
[70] A. Vermeij, T. C. Furfaro, and J. Alves, "NEMO: An architecture for software communications research in the maritime domain,” in Proc. MTS/IEEE Oceans, Genova, Italy, May 2015.

[71] NATO Standardization Agency, "ADatP-3(A) NATO message text formatting system (FORMETS), concept of FORMETS (CONFORMETS)," Brussels, Belgium, 2009, NATO/EAPC UNCLASSIFIED, Releasable to Australia, MD, ICI.

[72] “MOOS-IvP Home Page,” accessed: June 2016. [Online]. Available: http://www.moos-ivp.org/

[73] W. A. P. van Kleunen, N. A. Moseley, P. J. M. Havinga, and N. Meratnia, "Proteus II: Design and evaluation of an integrated power-efficient underwater sensor node," Hindawi Int. J. of Distributed Sensor Networks, Aug. 2015. [Online]. Available: http://dx.doi.org/10.1155/2015/791046

[74] W. van Kleunen, N. Moseley, N. Meratnia, and P. J. M. Havinga, "Experiments with aLS-Coop-Loc cooperative combined localization and time-synchronization," in Proc. WiNMeE, Hammamet, Tunisia, May 2014.

[75] EvoLogics, "Underwater acoustic modems," accessed: June 2016. [Online]. Available: https://evologics.de/en/products/ acoustics/index.html

[76] R. Otnes, "NILUS - An Underwater Acoustic Sensor Network Demonstrator System," in 10th Int. Mine Warfare Techn. Symp., Monterey, CA, USA, May 2012. [Online]. Available: http://www.ffi.no/no/Publikasjoner/Documents/NILUS2012.pdf

[77] Variscite, "VAR-SOM-OM44 V1.1 datasheet texas instruments OMAP4-based system-on-module," accessed: June 2016. [Online]. Available: http://www.variscite.com/images/stories/DataSheets/VAR-SOM-OM44_v1.1_datasheet_v1.24.pdf

[78] Texas Instruments, "8-channel high-quality low-power audio codec for portable applications," accessed: June 2016. [Online]. Available: http://www.ti.com/lit/gpn/tw16040

[79] H. Dol, P. Casari, and T. van der Zwan, "Software-defined open-architecture modems: Historical review and the NILUS approach,” in Proc. UCOMMS, Sestri Levante, Italy, Sep. 2014.

[80] P. van Walree, H. Buen, and R. Otnes, "A performance comparison between DSSS, M-FSK, and frequency-division multiplexing in underwater acoustic channels," in Proc. UComms, Sestri Levante, Italy, Sep. 2014.

[81] P. A. van Walree and G. Leus, "Robust underwater telemetry with adaptive turbo multiband equalization," IEEE J. Ocean. Eng., vol. 34, no. 4, pp. 645-655, Oct. 2009.

[82] H. Dol, M. Colin, P. van Walree, and R. Otnes, "How smartphone industry made UComms easier: Moore's law goes underwater," in Proc. MTS/IEEE Oceans, Genova, Italy, May 2015.

[83] R. Otnes and S. Haavik, "Duplicate reduction with adaptive backoff for a flooding-based underwater network protocol," in Proc. MTS/IEEE Oceans, Bergen, Norway, Jun. 2013.

[84] R. Otnes, P. A. van Walree, H. Buen, and H. Song, "Underwater acoustic network simulation with lookup tables from physical-layer replay," IEEE J. Ocean. Eng., vol. 40, no. 4, pp. 822-840, Sep. 2015. 